\section{RSP}

http://www.rsp.fsp.usp.br/
Revista de Saúde Pública

\title{
Critérios pragmáticos da definição de near miss neonatal: um estudo comparativo
}

\author{
Pauline Lorena Kale', Maria Helena Prado de Mello Jorge", Ruy Laurenti"l,, Sandra Costa \\ Fonseca"', Kátia Silveira da Silva ${ }^{\mathrm{IV}}$
}

' Universidade Federal do Rio de Janeiro. Instituto de Estudos em Saúde Coletiva. Área de Epidemiologia e Bioestatística. Rio de Janeiro, RJ, Brasil

" Universidade de São Paulo. Faculdade de Saúde Pública. Departamento de Epidemiologia. São Paulo, SP, Brasil

III Universidade Federal Fluminense. Instituto de Saúde Coletiva. Departamento de Epidemiologia e Bioestatística. Niterói, RJ, Brasil

Iv Fundação Oswaldo Cruz. Instituto Nacional de Saúde da Mulher, da Criança e do Adolescente Fernandes Figueira. Departamento de Epidemiologia Clínica. Rio de Janeiro, RJ, Brasil

\section{RESUMO}

OBJETIVO: Testar a validade dos critérios pragmáticos de definições de near miss neonatal, estendendo-as para todo o período infantil, e estimar indicadores de assistência perinatal em maternidades públicas.

MÉTODOS: Constituiu-se uma coorte de nascidos vivos de seis maternidades dos municípios de São Paulo, Niterói e Rio de Janeiro em 2011. Foram realizadas entrevistas e consultas aos cartões de pré-natal e prontuários. Critérios pragmáticos (peso ao nascer, idade gestacional e escore de Apgar 5’) das definições de near miss de Pileggi et al., Pileggi-Castro et al., Souza et al. e Silva et al. foram comparados. Foram calculados sensibilidade, especificidade (padrão-ouro: óbito infantil), percentual de óbitos entre recém-nascidos com ameaça à vida e taxas de near miss, mortalidade e desfechos graves por 1.000 nascidos vivos.

* In memoriam

Correspondência:

Pauline Lorena Kale.

Instituto de Estudos em Saúde

Coletiva - UFRJ

Av. Horácio Macedo, s/n

Cidade Universitária

21941-598 Rio de Janeiro,

RJ, Brasil

E-mail: pkale@iesc.ufrj.br

Recebido: 30 jul 2015

Aprovado: 15 nov 2016

Como citar: Kale PL, Jorge MHPM Laurenti R, Fonseca SC, Silva KS. Critérios pragmáticos da definição de near miss neonatal: um estudo comparativo. Rev Saude Publica. 2017:51:111.

Copyright: Este é um artigo de acesso aberto distribuído sob os termos da Licença de Atribuição Creative Commons, que permite uso irrestrito, distribuição e reprodução em qualquer meio, desde que o autor e a fonte originais sejam creditados.

RESULTADOS: Foram analisados 7.315 recém-nascidos (completude das informações > 99\%). A sensibilidade da definição de Pileggi-Castro et al. foi maior, resultando em um maior número de casos de near miss; a de Souza et al. apresentou menor valor, e as de Pileggi et al. e de Silva et al. apresentaram valores intermediários. Há um aumento da sensibilidade quando se estende o período de 0-6 para 0-27 dias, e um declínio quando se amplia para 0-364 dias. Especificidades foram elevadas ( $\geq 97 \%$ ) e superiores às sensibilidades (54\% a 77\%). Uma maternidade de São Paulo e outra de Niterói apresentaram, respectivamente, as menores e as maiores: taxas de mortalidade infantil, near miss, e frequência de nascimentos em situação de ameaça à vida, independentemente da definição.

CONCLUSÕES: As definições de near miss baseadas exclusivamente em critérios pragmáticos são válidas e podem ser utilizadas para fins de monitoramento. Com base na literatura perinatal, os pontos de corte adotados por Silva et al. foram mais adequados. Estudos periódicos poderiam aplicar uma definição mais completa, com incorporação de critérios clínicos, laboratoriais e de manejo, incluindo as anomalias congênitas preditoras do óbito infantil.

DESCRITORES: Near miss, classificação. Mortalidade Infantil. Peso ao Nascer, Recém-nascido Prematuro e Índice de Apgar. 


\section{INTRODUÇÃO}

O recém-nascido que apresentou uma complicação grave nos primeiros dias de vida, quase morreu, mas sobreviveu durante o período neonatal é um caso de near miss neonatal ${ }^{13}$. Ainda não existe uma definição padrão dos critérios que classificam esse evento. Em analogia à definição do near miss materno ${ }^{19}$, identificam-se "complicações graves" - condições que ameaçam a vida do neonato -, e a sobrevivência a essas condições define o near miss neonatal ${ }^{13,14,16}$.

Apesar do declínio da mortalidade infantil no Brasil, em 2014 foram registrados 26.723 óbitos no período neonatal ${ }^{\text {a }}$. Considerando que ocorrem cerca de quatro casos de near miss para um óbito neonatal, a aplicação da definição de near miss permitirá identificar um maior contingente de nascimentos de risco ${ }^{2}$, o que representa uma vantagem para os estudos de fatores associados, por aumentar o poder ${ }^{2,16}$, particularmente quando a mortalidade neonatal é reduzida ${ }^{2}$.

Com base no conceito de near miss neonatal, também podem ser estimados indicadores que expressam a carga de recém-nascidos de risco (morbidade grave e mortalidade), subsidiando tanto o cálculo dos recursos necessários aos serviços de saúde quanto a avaliação da qualidade do atendimento prestado ${ }^{13,20}$.

A Organização Mundial da Saúde (OMS) propôs um protocolo com vários critérios como marcadores de near miss neonatal, classificados em categorias de indicadores de disfunção orgânica, clínicos ou laboratoriais, indicadores de manejo de gravidade e condições dos recém-nascidos - peso, idade gestacional e asfixia - associadas à maior gravidade no período neonatal ${ }^{20}$.

Baixo peso, prematuridade e asfixia ao nascer são condições fortemente associadas aos óbitos neonatais ${ }^{2,9}$, e diferentes pontos de corte de peso ao nascer, idade gestacional e escore de Apgar no quinto minuto vêm sendo estudados na composição de critérios pragmáticos para a definição de "ameaça à vida" e, posteriormente, de near miss neonatal ${ }^{13,14,16,20}$. São considerados pragmáticos pela ampla disponibilidade de informação em documentos médicos e bancos de dados do setor saúde ${ }^{13}$, além de terem sido validados, associados ou não a outros critérios, para identificar near miss neonatal ${ }^{13,14,16}$.

Pileggi et al. ${ }^{13}$ validaram uma definição pragmática e operacional de near miss neonatal em maternidades brasileiras ${ }^{\mathrm{b}}$, considerando apenas informações sobre idade gestacional, peso ao nascer e escore de Apgar no quinto minuto. Dados de dois grandes inquéritos da OMS foram utilizados para validar a definição de near miss neonatal, comparando critérios pragmáticos das definições anteriormente mencionadas e incluindo em uma nova definição, além das condições do recém-nascido, os marcadores de manejo ${ }^{14}$. O estudo Nascer no Brasil, de base hospitalar e representatividade nacional, validou a definição de near miss neonatal, incluindo marcadores de condições dos recém-nascidos, uso de ventilação mecânica e presença de anomalia congênita ${ }^{16}$. As diferenças entre os critérios baseados no peso ao nascer, na idade gestacional e no escore de Apgar no quinto minuto das quatro definições de near miss dos

a Ministério da Saúde (BR), Secretaria de Vigilância em Saúde, Departamento de DST, Aids e Hepatites Virais. Painel de monitoramento da mortalidade infantil. Brasília (DF); 2017 [citado 16 jun 2017]. Disponível em: http://svs.aids.gov.br/ dashboard/mortalidade/infantil. show.mtw

${ }^{b}$ World Health Organization. Human Reproduction Programme: global survey on maternal and perinatal health. Geneva; c2017 [citado 16 jun 2017]. Disponível em: http:// www.who.int/reproductivehealth/ topics/maternal_perinatal/ globalsurvey/en/ estudos mencionados são os pontos de cortes. Somente o estudo de Silva et al. ${ }^{16}$ analisou todo o período neonatal (0-27 dias), e não apenas o período neonatal precoce (0-6 dias).

A identificação do conjunto de critérios pragmáticos que melhor capta os neonatos com morbidades neonatais graves (condições que ameaçam à vida) possibilita a aplicação desses critérios em contextos socioeconômicos diferenciados e na indisponibilidade de tecnologias aplicadas à assistência.

Para que o conceito de near miss possa ser um instrumento efetivo para melhoria da saúde materno-infantil, é necessário identificar casos de near miss e analisar os indicadores correspondentes ${ }^{20}$.

Neste artigo, testamos a validade dos critérios pragmáticos das definições existentes de near miss neonatal, estendendo-as para todo o período infantil e estimamos indicadores de assistência perinatal, relacionados ao conceito de near miss neonatal, em maternidades públicas. 


\section{MÉTODOS}

Foi constituída uma coorte de nascidos vivos (NV) a partir de um estudo transversal em maternidades do Sistema Único de Saúde (SUS) com alta frequência de nascidos vivos, nos municípios de São Paulo, SP, Niterói e Rio de Janeiro, RJ, em três meses do segundo semestre de 2011. As quatro entre as maiores maternidades paulistas são: uma filantrópica (A), única que não é referência para gravidez de alto risco; uma referência nacional em Saúde da Mulher e do recém-nascido (B); uma pertencente a hospital universitário (C); e outra referência para gravidez e parturiente de alto risco e neonatal na região Metropolitana da Grande São Paulo (D). A maternidade selecionada da cidade do Rio de Janeiro (E), localizada na Região Metropolitana I, assiste à clientela local, essencialmente da Área de Planejamento do município a que pertence, e a de Niterói (F) é referência para gravidez de baixo e alto risco da Região Metropolitana II do estado do Rio de Janeiro.

Este estudo é resultante da integração de pesquisas sobre a saúde de mulheres internadas por aborto ou para parto e seus conceptos nas cidades de São Paulo (Fapesp - Edital PPSUS 2009), do Rio de Janeiro e de Niterói (MS/Programa de Educação pelo Trabalho para a Saúde PET Saúde/Vigilância em Saúde - Edital 72010 e CNPq - Edital 20/2010). O instrumento principal para coleta de dados e a logística do estudo foram comuns.

A coleta de dados primários foi realizada por meio de entrevista à parturiente após 12 horas do parto por estudantes da área da saúde previamente treinados. Adicionalmente, foram consultados prontuários hospitalares, cartão da gestante e registro de sala de parto. Particularmente as informações sobre peso ao nascer e escore de Apgar foram obtidas em prontuários hospitalares. Nas maternidades de São Paulo, a informação da idade gestacional foi obtida fundamentalmente pelo prontuário hospitalar sem menção ao método de cálculo ou à estimativa, seguindo protocolos próprios dos médicos que assistiram às parturientes. No Rio de Janeiro, o método de cálculo ou estimativa da idade gestacional seguiu um algoritmo que priorizou as diferentes fontes de dados, nesta ordem: a data da última menstruação (DUM), quando compatível com a ultrassonografia (USG) realizada antes de 20 semanas; a USG, quando os valores da DUM eram incompatíveis ou ignorados; por último, o exame clínico neonatal. A informação foi trabalhada em semanas completas de gestação.

Foram selecionados para análise $7.362 \mathrm{NV}$, sendo excluído cerca de $1 \%$ dos registros por não apresentarem alguma das informações: peso ao nascer, idade gestacional ou escore de Apgar no quinto minuto. A população de estudo foi, portanto, composta por $7.315 \mathrm{NV}$, sendo 5.535, 1.222 e 557 provenientes, respectivamente, das maternidades das cidades de São Paulo, do Rio de Janeiro e de Niterói.

Informações sobre óbitos infantis ocorridos após alta hospitalar foram obtidas pelo Programa de Aprimoramento das Informações de Mortalidade (PRO-AIM), da Prefeitura do Município de São Paulo e pelo Sistema de Informações sobre Mortalidade (SIM) da Secretaria de Estado de Saúde do Rio de Janeiro (relacionamento probabilístico das bases de dados).

Os critérios pragmáticos marcadores de condições de ameaça à vida do neonato e os pontos de corte que embasam cada uma das quatro definições de near miss selecionadas baseiam-se em: peso ao nascer, idade gestacional e escore de Apgar no quinto minuto (Tabela 1).

Tabela 1. Situações que ameaçam a vida dos recém-nascidos: critérios pragmáticos de definições de near miss* neonatal.

\begin{tabular}{lccc}
\hline Definições de near miss* & \multicolumn{3}{c}{ Critérios pragmáticos } \\
\cline { 2 - 4 } & $\begin{array}{c}\text { Peso ao nascer } \\
\text { (gramas) }\end{array}$ & $\begin{array}{c}\text { Idade gestacional } \\
\text { (semanas) }\end{array}$ & $\begin{array}{c}\text { Escore de Apgar no } \\
\text { quinto minuto }\end{array}$ \\
\hline Pileggi et al. ${ }^{13}$ & $<1.500$ & $<30$ & $<7$ \\
${\text { Pileggi-Castro et al. }{ }^{14}}_{\text {Souza et al. }^{20}}^{<1.750}$ & $<33$ & $<7$ \\
Silva et al. ${ }^{16}$ & $<1.500$ & $<31$ & $<5$ \\
\hline
\end{tabular}

* As definições de near miss serão denominadas nesse estudo comparativo pelo sobrenome do primeiro autor. 
A validação da definição de near miss neonatal com dados nacionais foi realizada respectivamente por Pileggi et al. ${ }^{13}$ (0-6 dias) e Silva et al. ${ }^{16}$ (0-27 dias). O processo de validação, independentemente dos critérios, usa os óbitos neonatais como padrão-ouro.

Aúnica definição de near miss baseada exclusivamente nos critérios pragmáticosédePileggi et al. ${ }^{13}$ As demais incluem critérios clínicos, laboratoriais e de manejo, e a de Silva et al. ${ }^{16}$ inclui, ainda, a presença de anomalias congênitas. Quando diferentes pontos de corte são comparados, a escolha recai sobre a combinação de maior sensibilidade e menor heterogeneidade ${ }^{14}$. Apenas Souza et al. ${ }^{20}$ apresentam a definição de near miss sem o correspondente estudo de validação.

No presente estudo, foram analisados apenas os critérios pragmáticos de cada definição de near miss por componente etário infantil (neonatal precoce: 0-6, neonatal: 0-27 e infantil: 0-364 dias completos).

Foram calculadas as frequências das quatro definições avaliadas de ameaça à vida (ter pelo menos um dos critérios pragmáticos presentes). A população de estudo foi descrita em relação às situações que ameaçam à vida dos recém-nascidos, morbidade grave (near miss) e mortalidade infantil. Adicionalmente, foram estimadas as frequências de informações faltantes ou ignoradas das variáveis: peso ao nascer, idade gestacional e escore de Apgar no quinto minuto.

Para a validação das definições de near miss baseadas somente nos critérios pragmáticos, foram calculadas sensibilidade, especificidade (considerando como padrão-ouro o óbito infantil por componente etário) e os respectivos intervalos de confiança (IC95\%). Em relação à escolha do melhor conjunto de critérios, foi avaliada a combinação de sensibilidade e especificidade, o desempenho na avaliação da qualidade da assistência e, com base na literatura, a adequação dos pontos de corte utilizados em perinatologia. As definições de near miss foram denominadas nesse estudo comparativo pelo sobrenome do primeiro autor.

Considerando a proposta de Pileggi et al. ${ }^{13}$, adaptada do near miss materno da OMS, foram calculados os seguintes indicadores por maternidade e total:a) Para avaliar a carga de morbidade grave e de mortalidade assistidas nas maternidades no momento do nascimento: taxas de near miss (número de neonatos que atendem a qualquer um dos critérios validados, mas que sobreviveram), de mortalidade neonatal precoce e de desfechos graves (somatório dos casos de near miss e óbitos) por $1.000 \mathrm{NV}$ e b) Para avaliação da necessidade de investimento na melhor assistência perinatal: percentual de óbitos infantis por componentes entre os casos de neonatos com condições de ameaça à vida, que expressa a letalidade. Foram calculadas as taxas de mortalidade infantil neonatal e infantil e a razão entre as taxas de near miss e de mortalidade. O cálculo de todos os indicadores foi estendido para 0-27 e 0-364 dias, segundo as definições de near miss que, na análise anterior, mostraram-se válidas e adequadas. Os dados foram analisados pelo pacote estatístico SPSS ${ }^{\circledR}$, versão 17 e em Planilha eletrônica do programa Excel.

Este estudo foi aprovado pelo Comitê de Ética em Pesquisa do Instituto de Estudos em Saúde Coletiva da Universidade Federal do Rio de Janeiro (Processo 15/2010) e da Faculdade de Saúde Pública da Universidade de São Paulo (Processo 2188/11), da Secretaria de Saúde e Defesa Civil do Município do Rio de Janeiro (Processo 87/2011) e dos hospitais participantes.

\section{RESULTADOS}

Na população elegível para análise, $7.362 \mathrm{NV}$, a informação sobre o peso ao nascer esteve presente em todos os registros da pesquisa (Tabela 2). A idade gestacional foi a informação com maior frequência de incompletude, seguida do escore de Apgar, embora as magnitudes sejam inferiores a $1 \%$. Como esperado, critérios pragmáticos com pontos de corte mais elevados aumentaram a frequência de recém-nascidos vivos em situação de ameaça à vida. Na maternidade de Niterói, nasceram mais crianças nessa situação, independentemente de qual das quatro definições de near miss foi utilizada para identificar a presença de pelo menos um critério pragmático. Os menores percentuais de recém-nascidos em situação de ameaça à vida corresponderam às maternidades de São Paulo (Tabela 2). 
A seguir, foram analisados 7.315 NV que apresentavam as três informações: peso ao nascer, idade gestacional e escore de Apgar no quinto minuto.

O ranking de frequência dos critérios pragmáticos isolados e suas possíveis combinações para caracterização de situação de ameaça à vida mostraram variação, segundo as definições de near miss (Figura). Em todas as definições, exceto na de Souza et al. ${ }^{20}$, a soma dos critérios isolados foi superior a 50\%. A combinação dos critérios baseados no peso ao nascer e na idade gestacional classificou com maior frequência recém-nascidos

Tabela 2. Frequência de critérios pragmáticos de ameaça à vida e da presença de pelo menos um dos critérios segundo definições estudadas ${ }^{a}$. Municípios de São Paulo, Rio de Janeiro e Niterói, 2011.

\begin{tabular}{|c|c|c|c|c|c|c|c|c|}
\hline \multirow{4}{*}{$\begin{array}{l}\text { Situação de ameaça à vida } \\
\text { Critérios pragmáticos } \\
\end{array}$} & \multicolumn{8}{|c|}{ Cidade de localização das maternidades públicas } \\
\hline & \multirow{2}{*}{\multicolumn{2}{|c|}{$\begin{array}{l}\text { São Paulo } \\
n=5.579 \\
\end{array}$}} & \multirow{2}{*}{\multicolumn{2}{|c|}{$\begin{array}{c}\begin{array}{c}\text { Rio de } \\
\text { Janeiro }\end{array} \\
\mathrm{n}=1.224\end{array}$}} & \multirow{2}{*}{\multicolumn{2}{|c|}{$\begin{array}{l}\text { Niterói } \\
\mathbf{n}=559\end{array}$}} & \multirow{2}{*}{\multicolumn{2}{|c|}{$\begin{array}{c}\text { Total } \\
\mathrm{n}=7.362\end{array}$}} \\
\hline & & & & & & & & \\
\hline & $\mathrm{n}$ & $\%$ & $\mathrm{n}$ & $\%$ & $\mathrm{n}$ & $\%$ & $\mathrm{n}$ & $\%$ \\
\hline \multicolumn{9}{|l|}{ Peso ao nascer (PN-gramas) } \\
\hline$<1.500 \mathrm{~g}$ & 56 & 1,0 & 17 & 1,4 & 17 & 3,0 & 90 & 1,2 \\
\hline$<1.750 \mathrm{~g}$ & 98 & 1,8 & 28 & 2,3 & 29 & 5,2 & 155 & 2,1 \\
\hline Ignorado & 0 & - & 0 & - & 0 & - & 0 & - \\
\hline \multicolumn{9}{|l|}{ Idade gestacional (IG-semanas) } \\
\hline$<30 \mathrm{~s}$ & 45 & 0,8 & 11 & 0,9 & 11 & 2,0 & 67 & 0,9 \\
\hline$<31 \mathrm{~s}$ & 56 & 1,0 & 14 & 1,1 & 15 & 2,7 & 85 & 1,2 \\
\hline$<32 \mathrm{~s}$ & 70 & 1,3 & 20 & 1,6 & 18 & 3,2 & 108 & 1,5 \\
\hline$<33 \mathrm{~s}$ & 97 & 1,7 & 28 & 2,3 & 26 & 4,7 & 151 & 2,1 \\
\hline Ignorada & 27 & 0,5 & 0 & - & 0 & - & 27 & 0,4 \\
\hline \multicolumn{9}{|l|}{ Escore de Apgar 5' } \\
\hline$<5$ & 11 & 0,2 & 5 & 0,4 & 8 & 1,4 & 24 & 0,3 \\
\hline$<7$ & 44 & 0,8 & 19 & 1,6 & 15 & 2,7 & 78 & 1,0 \\
\hline Ignorado & 18 & 0,3 & 2 & 0,2 & 2 & 0,4 & 22 & 0,3 \\
\hline \multicolumn{9}{|l|}{ Pelo menos um dos critérios pragmáticos } \\
\hline $\mathrm{PN}<1.500 \mathrm{~g}$ ou IG $<30$ s ou escore de Apgar $5^{\prime}<7^{\mathrm{b}}$ & 93 & 1,7 & 34 & 2,8 & 23 & 4,1 & 150 & 2,0 \\
\hline $\mathrm{PN}<1.750 \mathrm{~g}$ ou IG $<33$ s ou escore de Apgar $5^{\prime}<7^{c}$ & 152 & 2,7 & 50 & 4,1 & 39 & 7,0 & 241 & 3,3 \\
\hline $\mathrm{PN}<1.500 \mathrm{~g}$ ou IG $<31$ s ou escore de Apgar $5^{\prime}<5^{\mathrm{d}}$ & 73 & 1,3 & 24 & 2,0 & 20 & 3,6 & 117 & 1,6 \\
\hline $\mathrm{PN}<1.500 \mathrm{~g}$ ou IG $<32 \mathrm{~s}$ ou escore de Apgar $5^{\prime}<7^{\mathrm{e}}$ & 110 & 2,0 & 38 & 3,1 & 26 & 4,7 & 174 & 2,4 \\
\hline Ignorado & 43 & 0,8 & 2 & 0,2 & 2 & 0,4 & 47 & 0,6 \\
\hline
\end{tabular}

a As definições de near miss serão denominadas neste estudo comparativo pelo sobrenome do primeiro autor.

b Pileggi et al. ${ }^{13}$

c Pileggi-Castro et al. ${ }^{14}$

${ }^{\mathrm{d}}$ Souza et al. ${ }^{20}$

e Silva et al..$^{16}$

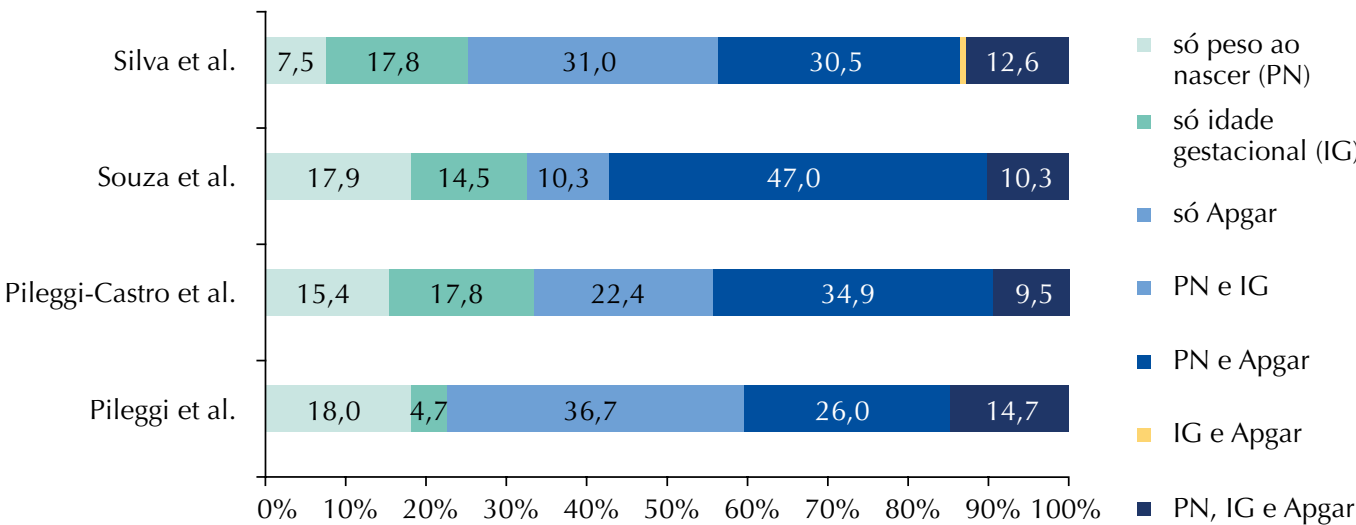

* Não houve caso de near miss para as combinações dos critérios pragmáticos PN e Apgar e na combinação de IG e Apgar, apenas na definição de Silva et al. ${ }^{16}(0,6 \%)$.

Pileggi et al. ${ }^{13}$ : peso ao nascer $(\mathrm{PN})<1.500 \mathrm{~g}$ ou idade gestacional $(\mathrm{IG})<30$ semanas ou escore de Apgar $5^{\prime}<7$. Pileggi-Castrol et al. ${ }^{14}$ : $\mathrm{PN}<1.750$ g ou IG $<33$ semanas ou escore de Apgar $5^{\prime}<7$.

Souza et al. ${ }^{20}: \mathrm{PN}<1.500 \mathrm{~g}$ ou IG $<31$ semanas ou escore de Apgar $5^{\prime}<5$.

Silva et al. ${ }^{16}: \mathrm{PN}<1.500 \mathrm{~g}$ ou IG $<32$ semanas ou escore de Apgar $5^{\prime}<7$.

Figura. Frequência dos critérios pragmáticos e suas combinações* segundo definição de near miss neonatal. Municípios de São Paulo, Rio de Janeiro e Niterói, 2011. 
em situação de ameaça à vida, excetuando-se a definição de Pileggi et al. ${ }^{13}$, segundo a qual predominou o critério isolado baseado no escore de Apgar. Nenhum recém-nascido apresentou a combinação de escore de Apgar com peso ao nascer e de IG com escore de Apgar, apenas para a definição de Silva et al. ${ }^{16}(0,6 \%)$. Apresentar os três critérios pragmáticos ocorreu em menor número, independentemente da definição de near miss.

Na Tabela 3, apresentam-se as frequências de recém-nascidos em condição de ameaça à vida, de casos de near miss e mortalidade infantil por componentes e os indicadores de validação segundo definição de near miss.

Ao apresentar pelo menos um dos critérios pragmáticos da definição de near miss, 150 (2,1\%), 241 (3,3\%), $117(1,6 \%)$ e $174(2,4 \%)$ NV foram classificados como neonatos com ameaça à vida, segundo Pileggi et al..$^{13}$, Pileggi-Castro et al. ${ }^{14}$, Souza et al. ${ }^{20}$ e Silva et al. ${ }^{16}$, respectivamente (Tabela 3 ).

Ocorreram 34 óbitos neonatais precoces, 13 neonatais tardios e 21 pós-neonatais. Independentemente do componente etário infantil, a sensibilidade da definição de near miss de Pileggi-Castro et al. ${ }^{14}$ foi maior e, consequentemente, resultou em maior número de falsos positivos (NV com risco e sobreviventes) (Tabela 3). Como a definição de near miss de Souza et al..$^{20}$ apresentou menor valor de sensibilidade, classificou um menor número de nascidos vivos como caso de near miss. As definições de near miss de Pileggi et al. ${ }^{13} \mathrm{e}$ de Silva et al. ${ }^{16}$ apresentaram valores próximos de sensibilidade (Tabela 3). Para as quatro definições, a sensibilidade permaneceu igual ou aumentou quando se estendeu o período de sobrevivência até 27 dias completos e diminuiu quando se estendeu até menor de um ano de idade. O maior valor de sensibilidade correspondeu à definição de Pileggi-Castro et al. ${ }^{14}$ no período neonatal $(76,6 \%)$, e o menor à definição de Souza et al. ${ }^{20}$ entre os menores de um ano de idade $(54,4 \%)$. Os valores de especificidade foram elevados $(\geq 97 \%)$ e superiores aos de sensibilidade (aproximadamente entre $54 \%$ e $77 \%$ ). A sobreposição dos intervalos de confiança de sensibilidade e de especificidade sugere que não há diferença estatisticamente significante entre as quatro definições.

Os indicadores de nascimento e óbitos e de avaliação da assistência perinatal são apresentados na Tabela 4. A maternidade A (São Paulo) apresentou os melhores indicadores:

Tabela 3. Frequências de recém-nascido de risco, casos de near miss por componentes infantil, sensibilidade, especificidade e intervalos de confiança (IC95\%) segundo definição de near miss. Municípios de São Paulo, Rio de Janeiro e Niterói, 2011.

\begin{tabular}{|c|c|c|c|c|}
\hline \multirow{2}{*}{ Variável } & \multicolumn{4}{|c|}{ Critérios pragmáticos } \\
\hline & Pileggi et al. ${ }^{a}$ & Pileggi-Castro et al. ${ }^{b}$ & Souza et al.c & Silva et al. ${ }^{d}$ \\
\hline Nascidos vivos com ameaça à vida & 150 & 241 & 117 & 174 \\
\hline \multicolumn{5}{|l|}{ Neoprecoce $0-6$ dias } \\
\hline Casos de near miss & 125 & 215 & 94 & 149 \\
\hline Sensibilidade (\%; IC95\%) & $74,3(57,8-86,0)$ & $76,5(59,8-87.8)$ & $67,6(50,8-81,0)$ & $73,5(56,7-85,6)$ \\
\hline Especificidade (\%; IC95\%) & $98,3(98,0-98,6)$ & $97,0(96,6-97,4)$ & $98,7(98,4-99,0)$ & $98,0(97,6-98,3)$ \\
\hline \multicolumn{5}{|l|}{ Neonatal 0-27 dias } \\
\hline Casos de near miss & 116 & 205 & 85 & 140 \\
\hline Sensibilidade (\%; IC95\%) & $72,3(58,1-83,2)$ & $76,6(62,6-86,6)$ & $68,1(53,8-79,7)$ & $72,3(58,1-83,2)$ \\
\hline Especificidade (\%; IC95\%) & $98,4(98,1-98,7)$ & $97,2(96,8-97,56)$ & $98,8(98,6-99,1)$ & $98,1(97,7-98,4)$ \\
\hline \multicolumn{5}{|l|}{ Infantil $<1$ ano } \\
\hline Casos de near miss & 110 & 198 & 80 & 134 \\
\hline Sensibilidade (\%; IC95\%) & $58,8(47,0-69,8)$ & $63,2(51,3-73,7)$ & $54,4(42,7-65,7)$ & $58,8(47,0-69,8)$ \\
\hline Especificidade (\%; IC95\%) & $98,5(98,2-98,7)$ & $97,3(96,9-97,6)$ & $98,9(98,6-99,1)$ & $98,2(97,8-98,4)$ \\
\hline
\end{tabular}


menores taxas de mortalidade infantil e menor número de nascimentos em situação de ameaça à vida, de taxas de near miss e desfechos graves, independentemente da definição de near miss. No outro extremo, a maternidade F (Niterói) apresentou a pior situação segundo os indicadores analisados. Destaca-se que, nessa maternidade, mais da metade dos recém-nascidos em situação de ameaça à vida segundo a definição de near miss de Pileggi et al. ${ }^{13}$ morreu antes de completar um ano de idade (Tabela 4).

Utilizando a definição de Silva et al. ${ }^{16}$, aproximadamente, para cada três sobreviventes em situação de ameaça à vida, ocorre um óbito neonatal na maternidade A (SP). Essa razão eleva-se para 3,6, 4,6 e 6,0 nas maternidades B, D e C (SP), respectivamente. No estado do Rio de Janeiro, as duas cidades representadas no estudo apresentaram as situações extremas: na maternidade E (Rio

Tabela 4. Nascidos vivos e taxas de mortalidade por componente infantil e percentual de nascidos vivos com ameaça à vida, taxas de near miss neonatal, de desfecho grave (1.000 nascidos vivos), índice de mortalidade entre casos de near miss (\%) segundo definição de near miss e componente infantil. Municípios de São Paulo, Rio de Janeiro e Niterói, 2011.

\begin{tabular}{|c|c|c|c|c|c|c|c|c|}
\hline \multirow{3}{*}{ Near niss } & \multirow{3}{*}{ Indicador } & \multicolumn{6}{|c|}{ Cidade/Maternidade } & \multirow{3}{*}{ Total } \\
\hline & & \multicolumn{4}{|c|}{ São Paulo } & \multirow{2}{*}{$\begin{array}{c}\text { RJ } \\
E\end{array}$} & \multirow{2}{*}{$\frac{\text { Niterói }}{F}$} & \\
\hline & & A & B & $\mathrm{C}$ & D & & & \\
\hline \multirow[t]{4}{*}{ Total } & Nascidos vivos & 1.621 & 1.592 & 749 & 1.574 & 1.222 & 557 & 7.315 \\
\hline & Taxa de mortalidade $0-6$ dias & 1,9 & 6,9 & 2,7 & 4,4 & 2,5 & 14,4 & 4,1 \\
\hline & Taxa de mortalidade 0-27 dias & 2,5 & 8,8 & 2,7 & 5,7 & 3,3 & 25,1 & 6,4 \\
\hline & Taxa de mortalidade $<1$ ano & 4,3 & 11,9 & 5,3 & 10,2 & 5,7 & 26,9 & 9,3 \\
\hline Pileggi et al. ${ }^{a}$ & Nascidos vivos com ameaça à vida (\%) & 0,4 & 2,6 & 1,6 & 2,1 & 2,8 & 4,1 & 2,1 \\
\hline \multirow[t]{4}{*}{$0-6$ dias } & Casos de near miss & 6 & 35 & 10 & 26 & 31 & 17 & 125 \\
\hline & Taxa de near miss & 3,7 & 22,0 & 13,4 & 16,5 & 25,4 & 30,5 & 17,1 \\
\hline & Taxa de desfecho grave & 5,6 & 28,9 & 16,0 & 21,0 & 27,8 & 44,9 & 21,7 \\
\hline & $\begin{array}{l}\text { Óbitos entre recém-nascidos com } \\
\text { ameaça à vida (\%) }\end{array}$ & 14,3 & 14,6 & 16,7 & 21,2 & 8,8 & 26,1 & 16,7 \\
\hline \multirow[t]{4}{*}{$0-27$ dias } & Casos de near miss & 6 & 33 & 10 & 25 & 30 & 12 & 116 \\
\hline & Taxa de near miss & 3,7 & 20,7 & 13,4 & 15,9 & 24,5 & 21,5 & 15,9 \\
\hline & Taxa de desfecho grave & 6,2 & 29,5 & 16,0 & 21,6 & 27,8 & 46,7 & 22,3 \\
\hline & $\begin{array}{l}\text { Óbitos entre recém-nascidos com } \\
\text { ameaça à vida (\%) }\end{array}$ & 14,3 & 19,5 & 16,7 & 24,2 & 11,8 & 47,8 & 22,7 \\
\hline \multirow[t]{4}{*}{$<1$ ano } & Casos de near miss & 6 & 30 & 9 & 25 & 29 & 11 & 110 \\
\hline & Taxa de near miss & 3,7 & 18,8 & 12,0 & 15,9 & 23,7 & 19,7 & 15,0 \\
\hline & Taxa de desfecho grave & 8,0 & 30,8 & 17,4 & 26,0 & 29,5 & 46,7 & 24,3 \\
\hline & $\begin{array}{l}\text { Óbitos entre recém-nascidos com } \\
\text { ameaça à vida (\%) }\end{array}$ & 14,3 & 26,8 & 25,0 & 24,2 & 14,7 & 52,2 & 26,7 \\
\hline Silva et al. ${ }^{b}$ & Nascidos vivos com ameaça à vida (\%) & 0,7 & 2,9 & 1,9 & 2,5 & 3,1 & 4,7 & 2,4 \\
\hline \multirow[t]{4}{*}{$0-6$ dias } & Casos de near miss & 10 & 40 & 12 & 32 & 35 & 20 & 149 \\
\hline & Taxa de near miss & 6,2 & 25,1 & 16,0 & 20,3 & 28,6 & 35,9 & 20,4 \\
\hline & Taxa de desfecho grave & 8,0 & 32,0 & 18,7 & 24,8 & 31,1 & 50,3 & 25,0 \\
\hline & $\begin{array}{l}\text { Óbitos entre recém-nascidos com } \\
\text { ameaça à vida }(\%)\end{array}$ & 9,1 & 13,0 & 14,3 & 17,9 & 7,9 & 23,1 & 14,4 \\
\hline \multirow[t]{4}{*}{$0-27$ dias } & Casos de near miss & 10 & 38 & 12 & 31 & 34 & 15 & 140 \\
\hline & Taxa de near miss & 6,2 & 23,9 & 16,0 & 19,7 & 27,8 & 26,9 & 19,1 \\
\hline & Taxa de desfecho grave & 8,6 & 32,7 & 18,7 & 25,4 & 31,1 & 52,1 & 25,4 \\
\hline & $\begin{array}{l}\text { Óbitos entre recém-nascidos com } \\
\text { ameaça à vida }(\%)\end{array}$ & 9,1 & 17,4 & 14,3 & 20,5 & 10,5 & 42,3 & 19,5 \\
\hline \multirow[t]{4}{*}{$<1$ ano } & Casos de near miss & 10 & 35 & 11 & 31 & 33 & 14 & 134 \\
\hline & Taxa de near miss & 6,2 & 22,0 & 14,7 & 19,7 & 27,0 & 25,1 & 18,3 \\
\hline & Taxa de desfecho grave & 10,5 & 33,9 & 20,0 & 29,2 & 32,7 & 52,1 & 27,6 \\
\hline & $\begin{array}{l}\text { Óbitos entre recém-nascidos com } \\
\text { ameaça à vida (\%) }\end{array}$ & 9,1 & 23,9 & 21,4 & 20,5 & 13,2 & 46,2 & 23,0 \\
\hline
\end{tabular}

Pileggi et al. ${ }^{13}$ : peso ao nascer $(\mathrm{PN})<1.500 \mathrm{~g}$ ou idade gestacional $(\mathrm{IG})<30$ semanas ou escore de Apgar $5^{\prime}<7$.

${ }^{\text {b }}$ Silva et al. ${ }^{16}$ : $\mathrm{PN}<1.500 \mathrm{~g}$ ou IG $<32$ semanas ou escore de Apgar $5^{\prime}<7$. 
de Janeiro), o número de sobreviventes com ameaça de vida foi 11,7 vezes maior, enquanto na maternidade F (Niterói), foi apenas 2,5 vezes maior do que o número de óbitos neonatais.

\section{DISCUSSÃO}

A validação dos critérios pragmáticos das quatro definições de near miss em três diferentes componentes da mortalidade infantil, com os dados do presente estudo, mostrou valores semelhantes de sensibilidade e especificidade. Com base apenas nesses resultados, podemos inferir que não há diferença estatisticamente significante entre as quatro definições avaliadas.

Os pontos de corte dos critérios pragmáticos variaram segundo as definições de near miss analisadas, principalmente da idade gestacional. Baseados na força de associação com óbito neonatal, todos se apresentaram válidos nos estudos de near miss neonatal, inclusive no presente estudo. Entretanto, consideramos que o ponto de corte em 32 semanas (inclui as categorias de extremo pré-termo: $\leq 28$ semanas e muito pré-termo: $>28$ e $<32$ semanas $^{8}$ ) é o mais adequado, por basear-se em etiologias e intervenções distintas no curso da gestação ${ }^{18}$ e por ser utilizado com mais frequência do que os demais nos estudos epidemiológicos ${ }^{4,7,8,11}$ $(<30 ;<31$ e $<33)$. Em um estudo recente, por meio de análise sistemática de bancos de dados nacionais e artigos científicos, a estimativa mundial de pré-termo com menos de 32 semanas de 1990-2010 foi de 15,6\% ${ }^{4}$. Em países em desenvolvimento, crianças que nascem com o equivalente a 32 semanas têm poucas chances de sobrevivência e, em países desenvolvidos, a sobrevivência de pré-termo com 32 semanas ou mais é semelhante à de crianças a termo 3 .

Quanto ao peso ao nascer, apenas na definição de Pileggi-Castro et al..$^{14}$, o ponto de corte foi $1.750 \mathrm{~g}$, o que resultou em maior sensibilidade do critério, porém com um maior número de falsos positivos. O ponto de corte de $1.500 \mathrm{~g}$ e a classificação correspondente, muito baixo peso ao nascer, têm sido usados com mais frequência em estudos epidemiológicos e na classificação de risco perinatal ${ }^{8,17}$.

O escore do Apgar proposto há 60 anos, não obstante a possibilidade de incorporar a eficácia de medidas imediatas de ressuscitação entre o primeiro e o quinto minuto quando aferido no quinto minuto, continua sendo um forte preditor da mortalidade infantil, independentemente da presença de confundimentos e outros fatores de risco ${ }^{6}$. O ponto de corte cinco não é utilizado com frequência nos estudos epidemiológicos, pois o escore de Apgar analisado de forma dicotômica incluiria algum grau de asfixia na categoria de menor risco, levando à subestimação. Os intervalos mais usados são: entre sete e 10, considerado normal; entre zero e três, considerado baixo; e entre quatro e seis, considerado intermediário. O ponto de corte de cinco para o escore de Apgar no quinto minuto foi menos sensível e mais específico. No estudo de Pileggi-Castro et al. ${ }^{14}$, quando comparados os critérios pragmáticos das definições de near miss neonatal precoce, o critério de Souza et al. ${ }^{20}$, único com ponto de corte cinco para o escore de Apgar no quinto minuto, foi o menos sensível e o mais específico, como os nossos resultados. O ponto de corte sete mostrou-se mais adequado.

Estudos periódicos poderiam aplicar uma definição mais completa, baseando-se na literatura sobre o assunto e considerando a disponibilidade de maior tecnologia na assistência. Quando for possível obter informações qualificadas sobre critérios além dos pragmáticos, recomenda-se a incorporação dos critérios clínicos, laboratoriais e de manejo. A incorporação de anomalia congênita na definição de near miss deverá restringir-se às anomalias preditoras

'Ministério da Saúde (BR) Secretaria de Vigilância em Saúde, Departamento de Análise da Situação de Saúde. Manual de instruções para o preenchimento da Declaração de Nascido Vivo. Brasília (DF); 2011 [citado 16 jun 2017]. (Série A, Normas e Manuais Técnicos). Disponível em: http://www. saude.ms.gov.br/wp-content/ uploads/sites/88/2015/11/ inst_dn.pdf de mortalidade infantil neonatal, como as malformações graves do sistema nervoso central e cardiopatias congênitas, pois a maioria dos recém-nascidos com anomalias congênitas sobreviverá ${ }^{2}$ o que indica baixa especificidade desse critério. Além disso, alguma limitação em relação à complexidade do diagnóstico e ao momento de registro poderá prejudicar a obtenção da informação em maternidades. A informação sobre anomalias congênitas no Sistema de Informações sobre Nascidos Vivos (Sinasc) restringe-se às anomalias aparentes, verificadas pelo profissional responsável pelo parto ${ }^{c}$, sendo muitas vezes de baixo risco de morte neonatal, além de ser subestimada ${ }^{10}$. 
Quanto ao período de sobrevivência do recém-nascido das quatro definições de near miss analisadas, apenas a definição de Silva et al. ${ }^{16}$ referiu-se ao período neonatal de 0-27 dias. Os resultados do presente estudo mostraram, em geral, um aumento da sensibilidade da definição de near miss quando comparados os períodos de 0-6 e 0-27 dias completos e uma diminuição em relação ao período de 0-364 dias. Em situações nas quais o monitoramento de óbito infantil após a alta hospitalar não é possível, sugerimos restringir-se ao período neonatal precoce ou, caso contrário, adotar o período de 0-27 dias.

Os indicadores baseados nos critérios pragmáticos da definição de near miss neonatal precoce refletiram adequadamente a demanda assistida e a assistência intra-hospitalar na internação para parto, independentemente da definição de near miss neonatal. Recomendamos sua avaliação em conjunto, e não isoladamente.

Na maternidade F (Niterói), a carga de recém-nascidos graves (taxa de desfechos graves) e a proporção de óbitos entre os recém-nascidos com ameaça à vida, para o período de 0-6 dias completos, foram altas, respectivamente, 50,3\% NV e 23,1\% (definição de Silva et al. ${ }^{16}$ ). Considerando a alta demanda assistida de maior risco e de fora do município de localização da maternidade, esse resultado nos pareceu consistente, podendo refletir questões relacionadas à qualidade de atenção intra-hospitalar, o que deverá ser levado em consideração no momento de definir prioridades na assistência materno-infantil ${ }^{13}$. Contrastando com a maternidade F, como esperado, a maternidade A(SP) apresentou a menor carga de desfechos graves. Destaca-se que, na maternidade $\mathrm{E}(\mathrm{RJ})$, a carga de desfechos graves foi relativamente alta $(31,1 \% \mathrm{o})$, cerca de quatro vezes o valor da maternidade $A$, mas a mortalidade entre os recém-nascidos graves $(7,9 \%)$ foi a menor entre todas as maternidades avaliadas.

As principais vantagens dos critérios pragmáticos para definição de near miss são a disponibilidade das informações sobre peso ao nascer, idade gestacional e escore de Apgar nas próprias maternidades e pelo Sinasc e a facilidade de uso da classificação para fins clínicos e epidemiológicos. A completude das informações dos critérios pragmáticos neste estudo mostrou-se excelente ${ }^{15}$, ligeiramente superior a $99 \%$. Além da completude, trata-se de informações disponíveis e de fácil obtenção. A acurácia e a concordância de desfechos da gravidez (incluindo peso ao nascer, idade gestacional e escore de Apgar de informações dicotomizadas de prontuários médicos) costumam ser consideradas boas e não enviesadas ${ }^{1}$. Estudos nacionais mostraram que o peso ao nascer ${ }^{21} \mathrm{e}$ o escore de Apgar no quinto minuto apresentaram de boa a excelente confiabilidade ${ }^{5,21}$. A acurácia da idade gestacional, entretanto, pode ser prejudicada pelo próprio método de medida ${ }^{12, d}$. Apesar de não terem sido analisadas a acurácia e a confiabilidade dessas três variáveis em relação a diferentes fontes de informações nesta pesquisa, acredita-se que peso ao nascer e escore de Apgar no quinto minuto reproduzam o padrão de melhor validade e reprodutibilidade, se comparados à idade gestacional.

Tal como no estudo de Pileggi et al. ${ }^{13}$, nas maternidades de São Paulo, a informação da idade gestacional analisada foi obtida do prontuário hospitalar, que seguiu protocolos próprios dos médicos que assistiram às parturientes, sem menção ao método de cálculo ou estimativa utilizado. Nas duas maternidades do estado do Rio de Janeiro, os pesquisadores seguiram o algoritmo próprio, que priorizou a DUM, quando compatível com a USG realizada antes de 20 semanas, considerado o método mais válido ${ }^{12}$. Nesse sentido, a aferição da idade gestacional não foi padronizada.

Adicionalmente, indicadores de saúde baseados no near miss neonatal permitem monitorar a qualidade do atendimento prestado e da demanda assistida de um contingente de

d National Institute for Health and Care Excellence. Antenatal care: routine care for the healthy pregnant woman. London: RCOG Press; 2008 [citado 9 fev 2015]. (NICE Clinical Guideline, 62). Disponível em: http://www. nice.org.uk/nicemedia/pdf/ CG062NICEguideline.pdf nascimentos de risco, além de identificar crianças que demandarão maior atenção dos serviços de saúde e cuidado de seus familiares.

Uma limitação deste estudo foi a não aleatoriedade da amostra de maternidades e parturientes, embora o método utilizado seja semelhante ao da pesquisa da OMS ${ }^{19}$. Adicionalmente, as dificuldades em relação à maior precisão e à comparabilidade da idade gestacional são relatadas também em diversos estudos nacionais e internacionais ${ }^{8,12, d}$. 
Na medida em que a sensibilidade e a especificidade não mostraram diferenças expressivas, recomendamos a utilização da definição de Silva et al. ${ }^{16}$ devido à adequação dos pontos de corte com valores usados correntemente em estudos perinatais. Ademais, destacamos que essa definição de near miss neonatal baseou-se na análise de dados exclusivamente nacionais e, portanto, mais adequados à nossa realidade.

Recomendamos também que, sendo possível a obtenção da informação individualizada do Sinasc e do SIM, por exemplo, por meio do relacionamento probabilístico das respectivas bases de dados, ou no próprio hospital, os indicadores baseados no near miss sejam monitorados por estabelecimento de saúde, contribuindo para a avaliação da assistência materno-infantil.

\section{REFERÊNCIAS}

1. Ampt AJ, Ford JB, Taylor LK, Roberts CL. Are pregnancy outcomes associated with risk factor reporting in routinely collected perinatal data? NSW Public Health Bull. 2013;24(2):65-9. https://doi.org/10.1071/NB12116

2. Avenant T. Neonatal near miss: a measure of the quality of obstetric care. Best Pract Res Clin Obstet Gynaecol. 2009;23(3):369-74. https:doi.org/10.1016/j.bpobgyn.2008.12.005

3. Beck S, Wojdyla D, Say L, Betran AP, Merialdi M, Requejo JH, et al. The worldwide incidence of preterm birth: a systematic review of maternal mortality and morbidity. Bull World Health Organ. 2010 88(1):31-8. https://doi.org/10.2471/BLT.08.062554

4. Blencowe H, Cousens S, Oestergaard MZ, Chou D, Moller AB, Narwal R, et al. National, regional, and worldwide estimates of preterm birth rates in the year 2010 with time trends since 1990 for selected countries: a systematic analysis and implications. Lancet. 2012;379(9832):2162-72. https://doi.org/10.1016/S0140-6736(12)60820-4

5. Guimarães PV, Coeli CM, Cardoso RC, Medronho RA, Fonseca SC, Pinheiro RS. Reliability of data from a very low birth weight population in the Live Birth Information System 2005-2006. Rev Bras Epidemiol. 2012;15(4):694-704. https://doi.org/10.1590/S1415-790X2012000400002

6. Iliodromiti S, Mackay DF, Smith GC, Pell JP, Nelson SM. Apgar score and the risk of cause-specific infant mortality: a population-based cohort study. Lancet. 2014;384(9956):1749-55. https://doi.org/10.1016/S0140-6736(14)61135-1

7. Katz J, Lee AC, Kozuki N, Lawn JE, Cousens S, Blencowe $\mathrm{H}$, et al. Mortality risk in preterm and small-for-gestational-age infants in low-income and middle-income countries: a pooled country analysis. Lancet. 2013;382(9890):417-25. https://doi.org/10.1016/S0140-6736(13)60993-9

8. Lasswell SM, Barfield WD, Rochat RW, Blackmon L. Perinatal regionalization for very low-birth-weight and very preterm infants: a meta-analysis. JAMA. 2010;304(9):992-1000. https://doi.org/10.1001/jama.2010.1226

9. Lawn JE, Blencowe H, Oza S, You D, Lee AC, Waiswa P, et al. The Lancet Every Newborn Study Group. Every Newborn: progress, priorities, and potential beyond survival. Lancet. 2014;384(9938):189-205. https://10.1016/S0140-6736(14)60496-7

10. Luquetti DV, Koifman RJ. Qualidade da notificação de anomalias congênitas pelo Sistema de Informações sobre Nascidos Vivos (SINASC): estudo comparativo nos anos 2004 e 2007. Cad Saude Publica. 2010;26(9):1756-65. https://doi.org/10.1590/S0102-311X2010000900009

11. Moutquin JM. Classification and heterogeneity of preterm birth. BJOG. 2003;110 Suppl 20:30-3. https://doi.org/10.1046/j.1471-0528.2003.00021.X

12. Pereira APE, Dias MAB, Bastos MH, Gama SGN, Leal MC. Determining gestational age for public health care users in Brazil: comparison of methods and algorithm creation. BMC Res Notes. 2013;6:60. https://doi.org/10.1186/1756-0500-6-60

13. Pileggi C, Souza JP, Cecatti JG, Faúndes A. Neonatal near miss approach in the 2005 WHO Global Survey Brazil. J Pediatr (Rio J). 2010;86(1):21-6. https://doi.org/10.2223/JPED.1965

14. Pileggi-Castro C, Camelo Jr JS, Perdoná GC, Mussi-Pinhata MM, Cecatti JG, Mori $\mathrm{R}$, et al. Development of criteria for identifying neonatal near-miss cases: analysis of two WHO multicountry cross-sectional studies. BJOG. 2014;121 Suppl 1:110-8. https://doi.org/10.1111/1471-0528.12637 
15. Romero DE, Cunha CB. Avaliação da qualidade das variáveis epidemiológicas e demográficas do Sistema de Informações sobre Nascidos Vivos, 2002. Cad Saude Publica. 2007;23(3):701-14. https://doi.org/10.1590/S0102-311X2007000300028

16. Silva AAM, Leite AJM, Lamy ZC, Moreira MEL, Gurgel RQ, Cunha AJLA, et al. Morbidade neonatal near miss na pesquisa Nascer no Brasil. Cad Saude Publica. 2014;30 Supl 1:S182-91. https://doi.org/10.1590/0102-311X00129613

17. Silva $\mathrm{CH}$, Agranonik M, Silva AAM, Bettiol H, Barbieri MA, Goldani MZ. Secular trend of very low birth weight rate in Porto Alegre, Southern Brazil. J Biosoc Sci. 2010;42(2):243-53. https://doi.org/10.1017/S002193200999037X

18. Simmons LVE, Rubens CE, Darmstadt GL, Gravett MG. Preventing preterm birth and neonatal mortality: exploring the epidemiology, causes, and interventions. Semin Perinatol. 2010;34(6):408-15. https://doi.org/10.1053/j.semperi.2010.09.005

19. Souza JP, Cecatti JG, Faundes A, Morais SS, Villar J, Carroli G, et al. Maternal near miss and maternal death in the World Health Organization's 2005 global survey on maternal and perinatal health. Bull World Health Organ. 2010;88(2):113-9. https://doi.org/10.2471/BLT.08.057828

20. Souza JP, Gülmezoglu AM, Carroli G, Lumbiganon P, Qureshi Z; WHOMCS Research Group. The World Health Organization multicountry survey on maternal and newborn health: study protocol. BMC Health Serv Res. 2011;11:286. https://doi.org/10.1186/1472-6963-11-286

21. Theme Filha MM, Gama SGN, Cunha CB, Leal MC. Confiabilidade do Sistema de Informações sobre Nascidos Vivos Hospitalares no Município do Rio de Janeiro, 1999-2001. Cad Saude Publica. 2004;20 Supl 1:S83-91. https://doi.org/10.1590/S0102-311X2004000700009

Financiamento: Fundação de Amparo a Pesquisa do Estado de São Paulo (FAPESP - Edital PPSUS 2009, Processo 09/53253-8). Conselho Nacional de Desenvolvimento Científico e Tecnológico (CNPq - Edital/20/2010, Proceso 402426/2010-0). Instituto de Estudos em Saúde Coletiva (IESC-UFRJ - bolsas: PET Saúde/Vigilância em Saúde, contemplado pelo Edital 7 de 3 de março de 2010 do Ministério da Saúde e Secretaria Municipal de Saúde do Rio de Janeiro). Fundação Carlos Chagas Filho de Amparo à Pesquisa do Estado do Rio de Janeiro (FAPERJ Processo E-26/100.091/2012, bolsas de iniciação científica para Pires ACA) e Instituto de Saúde da Comunidade (ISC-UFF - bolsas de iniciação científica: CNPq e FAPERJ).

Contribuição dos Autores: Concepção e planejamento do estudo e supervisão da coleta de dados: RL. Concepção e planejamento do estudo, supervisão da coleta, análise e interpretação dos dados, elaboração do manuscrito, aprovação da versão final a ser publicada e assumem responsabilidade pública pelo conteúdo do artigo: PLK, MHMJ, SCF, KSS.

Conflito de Interesses: Os autores declaram não haver conflito de interesses. 